\title{
A new generalized harmonic evolution system
}

\author{
Lee Lindblom $^{1}$, Mark A Scheel ${ }^{1}$, Lawrence E Kidder ${ }^{2}$, Robert Owen ${ }^{1}$ \\ and Oliver Rinne ${ }^{1}$ \\ ${ }^{1}$ Theoretical Astrophysics 130-33, California Institute of Technology, Pasadena, CA 91125, USA \\ 2 Center for Radiophysics and Space Research, Cornell University, Ithaca, NY 14853, USA
}

Received 15 December 2005, in final form 8 February 2006

Published 27 July 2006

Online at stacks.iop.org/CQG/23/S447

\begin{abstract}
A new representation of the Einstein evolution equations is presented that is first order, linearly degenerate and symmetric hyperbolic. This new system uses the generalized harmonic method to specify the coordinates, and exponentially suppresses all small short-wavelength constraint violations. Physical and constraint-preserving boundary conditions are derived for this system, and numerical tests that demonstrate the effectiveness of the constraint suppression properties and the constraint-preserving boundary conditions are presented.
\end{abstract}

PACS numbers: $04.25 . \mathrm{Dm}, 04.20 . \mathrm{Cv}, 02.60 . \mathrm{Cb}$

\section{Introduction}

Harmonic and generalized harmonic $(\mathrm{GH})$ coordinates have played important roles in general relativity theory from the very beginning. Einstein used harmonic (then called isothermal) coordinates in his analysis of candidate theories of gravitation (as recorded in his Zurich notebook of 1912) before general relativity even existed [1], DeDonder used them to analyse the characteristic structure of general relativity in 1921 [2, 3], and Fock used them to analyse gravitational waves in 1955 [4]. Harmonic coordinates played an important role in the proofs of the well posedness of the Cauchy problem for the Einstein equations by Choquet-Bruhat in 1952 [5, 6] and by Fischer and Marsden in 1972 [7]. Harmonic coordinates have also been used to obtain numerical solutions of Einstein's equations by Garfinkle [8] and by Winicour and collaborators [9-11]. The idea of specifying arbitrary coordinate systems using a generalization of harmonic coordinates was introduced by Friedrich in 1985 [12]. And quite recently the $\mathrm{GH}$ approach to specifying coordinates played an important, perhaps seminal, role in the state-of-the-art numerical simulations of the final inspiral and merger of binary black-hole systems by Pretorius $[13,14]$ using a form of the equations suggested by Gundlach et al [15].

We think there are two important properties that have made harmonic or GH coordinates such an important tool throughout the history of general relativity theory. The first property is well known: this method of specifying the coordinates transforms the principal parts of 
the Einstein equations into a manifestly hyperbolic form, in which each component of the metric is acted on by the standard second-order wave operator. The second property is not as widely appreciated: this method of specifying coordinates fundamentally transforms the constraints of the theory. This new form of the constraints makes it possible to modify the evolution equations in a way that prevents small constraint violations from growing during numerical evolutions - without changing the physical solutions of the system and without changing the fundamental hyperbolic structure of the equations. The purpose of this paper is to explore and understand these important properties and to extend the GH evolution system in a way that makes it even more useful for numerical computations. In section 2 we review the modified form of the GH evolution system of Gundlach et al and Pretorius. We convert and extend this system in section 3 into a symmetric-hyperbolic first-order evolution system that has constraint suppression properties comparable to those of the second-order system. We derive and analyse the well posedness of constraint-preserving and physical boundary conditions for this new first-order system in section 4, and in section 5 we present numerical tests that demonstrate the effectiveness of its constraint suppression properties and the new constraint-preserving boundary conditions.

\section{Generalized harmonic evolution system}

Harmonic (sometimes called wave) coordinates are functions $x^{a}$ that satisfy the covariant scalar wave equation. These coordinates are very useful because they significantly simplify the second-derivative terms in the Ricci curvature tensor. To see this explicitly, consider a spacetime with metric tensor $\psi_{a b}$ :

$$
\mathrm{d} s^{2}=\psi_{a b} \mathrm{~d} x^{a} \mathrm{~d} x^{b}
$$

(We use Latin indices from the first part of the alphabet $a, b, c, \ldots$ to denote four-dimensional spacetime quantities.) A coordinate $x^{b}$ is called harmonic if it satisfies the scalar wave equation,

$$
0=\psi_{a b} \nabla^{c} \nabla_{c} x^{b}=-\Gamma_{a},
$$

where $\nabla_{c}$ denotes the covariant derivative compatible with $\psi_{a b}$, and $\Gamma_{a} \equiv \psi^{b c} \Gamma_{a b c}$ is the trace of the standard Christoffel symbol $\Gamma_{a b c}$ :

$$
\Gamma_{a b c}=\frac{1}{2}\left(\partial_{b} \psi_{a c}+\partial_{c} \psi_{a b}-\partial_{a} \psi_{b c}\right) .
$$

The right side of equation (2) is just the expression for this covariant wave operator acting on $x^{b}$ in terms of partial derivatives and Christoffel symbols.

The Ricci curvature tensor can be written as

$$
R_{a b}=-\frac{1}{2} \psi^{c d} \partial_{c} \partial_{d} \psi_{a b}+\nabla_{(a} \Gamma_{b)}+\psi^{c d} \psi^{e f}\left(\partial_{e} \psi_{c a} \partial_{f} \psi_{d b}-\Gamma_{a c e} \Gamma_{b d f}\right),
$$

in any coordinate system, where $\nabla_{a} \Gamma_{b} \equiv \partial_{a} \Gamma_{b}-\psi^{c d} \Gamma_{c a b} \Gamma_{d}$. In harmonic coordinates, $\Gamma_{a}=0$, so the only second-derivative term remaining in the Ricci tensor is $\psi^{c d} \partial_{c} \partial_{d} \psi_{a b}$. Therefore in harmonic coordinates the vacuum Einstein equations, $R_{a b}=0$, form a manifestly hyperbolic system [5],

$$
\psi^{c d} \partial_{c} \partial_{d} \psi_{a b}=2 \psi^{c d} \psi^{e f}\left(\partial_{e} \psi_{c a} \partial_{f} \psi_{d b}-\Gamma_{a c e} \Gamma_{b d f}\right)
$$

Friedrich [12] (and independently Garfinkle [8]) realized that the manifestly hyperbolic form of the Einstein system, equation (5), can also be achieved for arbitrary coordinates, if the choice of coordinates is fixed in a certain (but non-standard) way. This alternate method of specifying the choice of coordinates, which we call the generalized harmonic $(\mathrm{GH})$ method, is implemented by assuming that the coordinates satisfy the inhomogeneous wave equation,

$$
H_{a}(x, \psi)=\psi_{a b} \nabla_{c} \nabla^{c} x^{b}=-\Gamma_{a},
$$


where $H_{a}(x, \psi)$ is an arbitrary but fixed algebraic function of the coordinates $x^{a}$ and the metric $\psi_{a b}$ (but not its derivatives). In these $\mathrm{GH}$ coordinates $H_{a}=-\Gamma_{a}$, so the vacuum Einstein equations are again manifestly hyperbolic:

$$
\psi^{c d} \partial_{c} \partial_{d} \psi_{a b}=-2 \nabla_{(a} H_{b)}+2 \psi^{c d} \psi^{e f}\left(\partial_{e} \psi_{c a} \partial_{f} \psi_{d b}-\Gamma_{a c e} \Gamma_{b d f}\right) .
$$

The term containing $H_{b}$ on the right side of equation (7) is a pre-specified algebraic function ( of $x^{a}$ and $\psi_{a b}$ ) that operates as a source term, rather than one of the principal terms containing second derivatives of $\psi_{a b}$. The principal (i.e., second-derivative) parts of this GH evolution system, equation (7), are therefore identical to those of the harmonic evolution system, equation (5).

To understand the GH method of specifying coordinates more clearly, it is helpful to compare it to the more traditional way of specifying coordinates with the lapse and the shift. To do this we introduce a foliation of the spacetime by spacelike hypersurfaces, and adopt a coordinate system, $\left\{t, x^{k}\right\}$, with the $t=$ constant surfaces being the leaves of this foliation. The traditional lapse $N$, shift $N^{k}$ and three-dimensional spatial metric $g_{i j}$ associated with this coordinate system are then defined by

$$
\mathrm{d} s^{2}=\psi_{a b} \mathrm{~d} x^{a} \mathrm{~d} x^{b}=-N^{2} \mathrm{~d} t^{2}+g_{i j}\left(\mathrm{~d} x^{i}+N^{i} \mathrm{~d} t\right)\left(\mathrm{d} x^{j}+N^{j} \mathrm{~d} t\right) .
$$

(We use Latin indices $i, j, k, \ldots$ to denote three-dimensional spatial quantities; while Latin indices from the first part of the alphabet $a, b, c, \ldots$ will continue to denote four-dimensional quantities.) Expressing the GH coordinate condition, equation (6), in this $3+1$ language implies evolution equations for the lapse and shift:

$$
\begin{aligned}
& \partial_{t} N-N^{k} \partial_{k} N=-N\left(H_{t}-N^{i} H_{i}+N K\right), \\
& \partial_{t} N^{i}-N^{k} \partial_{k} N^{i}=N g^{i j}\left[N\left(H_{j}+g^{k l} \Gamma_{j k l}\right)-\partial_{j} N\right],
\end{aligned}
$$

where $K$ is the trace of the extrinsic curvature. Specifying the GH gauge function $H_{a}(x, \psi)$ therefore determines the time derivatives of the lapse $N$ and shift $N^{k}$, and hence the evolution of the gauge degrees of freedom of the system. Some gauge conditions (e.g., $N=1, N^{k}=0$ ) may not be simple conditions on $H_{a}$, just as some gauge conditions (e.g., $H_{a}=0$ ) are not simple conditions on $N$ and $N^{k}$. In this paper we restrict attention to the cases where $H_{a}(x, \psi)$ is a specified algebraic function. Any chosen coordinates can clearly be described (ex post facto) by an $H_{a}$ of this form. But $H_{a}$ may also be specified in more general ways, e.g., by giving evolution equations for $H_{a}$ [13]. We expect (but have not proved) that any coordinates can be obtained by specifying a priori suitable (possibly complicated) conditions on $H_{a}$.

\subsection{Constraint evolution}

Our experience in solving the Einstein equations numerically is that small constraint violations typically grow into large constraint violations that quickly make the solutions unphysical. We think it is essential therefore to understand the constraints and how violations of those constraints evolve with time. To this end it is helpful to consider the following representation of the GH system, equation (7):

$$
0=R_{a b}-\nabla_{(a} \mathcal{C}_{b)},
$$

where $R_{a b}$ is the Ricci tensor defined in equation (4), and $\mathcal{C}_{a}$ is defined as

$$
\mathcal{C}_{a}=H_{a}+\Gamma_{a}
$$

From this perspective the condition $\mathcal{C}_{a}=0$ serves as the constraint that ensures the coordinates satisfy the GH coordinate condition, equation (6). It is straightforward to verify that 
equation (11) is equivalent to the GH evolution equations, equation (7). This form of the GH system, equation (11), is also formally equivalent to the Z4 system [16] (in the sense that there is a one-to-one correspondence between solutions of the two systems), where the constraint $\mathcal{C}_{a}$ plays the role of the Z4 vector field [15]. The systems differ however in the way the fields are evolved: in the Z4 system the field $\mathcal{C}_{a}$ is evolved as a separate dynamical field, while in the $\mathrm{GH}$ representation $\mathcal{C}_{a}$ is treated as a constraint which is not evolved separately.

The evolution equation for the constraints is easily deduced from the GH evolution system, equation (11): take the divergence of the trace-reversed equation (11), use the contracted Bianchi identity $\nabla^{a} R_{a b}-\frac{1}{2} \nabla_{b} R=0$, and exchange the order of covariant derivatives with the Ricci identity, yielding

$$
0=\nabla^{b} \nabla_{b} \mathcal{C}_{a}+R_{a b} \mathcal{C}^{b}
$$

Finally the Ricci tensor can be eliminated using equation (11) to produce the following equation for the evolution of the constraints [17]:

$$
0=\nabla^{b} \nabla_{b} \mathcal{C}_{a}+\mathcal{C}^{b} \nabla_{(a} \mathcal{C}_{b)}
$$

This equation guarantees that the constraints $\mathcal{C}_{a}$ will remain zero within the domain of dependence of an initial surface on which $\mathcal{C}_{a}=\partial_{t} \mathcal{C}_{a}=0$. Thus the $\mathrm{GH}$ evolution system is self-consistent.

The standard Hamiltonian and momentum constraints of general relativity are encoded in the constraints of the GH system in an interesting way. Let $t^{a}$ denote the unit timelike normal to the $t=$ constant surfaces of the foliation used in equation (8). The standard Hamiltonian and momentum constraints are combined here into the single four-dimensional momentum constraint $\mathcal{M}_{a}$, which is given by the contraction of $t^{a}$ with the Einstein curvature tensor:

$$
\mathcal{M}_{a} \equiv\left(R_{a b}-\frac{1}{2} \psi_{a b} R\right) t^{b} .
$$

Using equation (11) for a spacetime that satisfies the GH evolution system, we see that

$$
t^{b} \nabla_{b} \mathcal{C}_{a}=2 \mathcal{M}_{a}+\left(g^{b c} t_{a}-t^{c} g^{b}{ }_{a}\right) \nabla_{b} \mathcal{C}_{c}
$$

where $g_{a b}=\psi_{a b}+t_{a} t_{b}$ is the intrinsic metric to the $t=$ constant hypersurfaces. Specifying the initial data needed to determine the evolution of the constraints, $\left\{\mathcal{C}_{a}, \partial_{t} \mathcal{C}_{a}\right\}$, via equation (14) is equivalent therefore to specifying the more usual representation of the constraints, $\left\{\mathcal{C}_{a}, \mathcal{M}_{a}\right\}$, on that surface.

\subsection{Constraint damping}

The impressive numerical simulations of binary black-hole spacetimes performed recently by Pretorius $[13,14]$ are based on a modified form of the GH evolution system suggested by Gundlach et al [15]. This modified system has the remarkable property that it causes constraint violations to be damped out as the system evolves. The modified system is given by

$$
0=R_{a b}-\nabla_{(a} \mathcal{C}_{b)}+\gamma_{0}\left[t_{(a} \mathcal{C}_{b)}-\frac{1}{2} \psi_{a b} t^{c} \mathcal{C}_{c}\right]
$$

where $t^{a}$ (as before) is the future directed timelike unit normal to the $t=$ constant surfaces, and $\gamma_{0}$ is a constant that determines the timescale on which the constraints are damped. This system can also be written more explicitly as

$$
\begin{aligned}
\psi^{c d} \partial_{c} \partial_{d} \psi_{a b}= & -2 \nabla_{(a} H_{b)}+2 \psi^{c d} \psi^{e f}\left(\partial_{e} \psi_{c a} \partial_{f} \psi_{d b}-\Gamma_{a c e} \Gamma_{b d f}\right) \\
& +\gamma_{0}\left[2 \delta^{c}{ }_{(a} t_{b)}-\psi_{a b} t^{c}\right]\left(H_{c}+\Gamma_{c}\right) .
\end{aligned}
$$

This system is manifestly hyperbolic since the additional constraint damping terms (i.e., those proportional to $\gamma_{0}$ ) do not modify the principal parts of the standard GH evolution system. It 
is also clear that the constraint-satisfying solutions of this system are identical to those of the standard Einstein system.

In order to understand how this modification affects the constraints, we must analyse the associated constraint evolution system. This can be done by following the same steps that lead to equation (13), but in this case we obtain

$$
0=\nabla^{b} \nabla_{b} \mathcal{C}_{a}+R_{a b} \mathcal{C}^{b}-2 \gamma_{0} \nabla^{b}\left[t_{(b} \mathcal{C}_{a)}\right]
$$

or using equation (17),

$$
0=\nabla^{c} \nabla_{c} \mathcal{C}_{a}-2 \gamma_{0} \nabla^{b}\left[t_{(b} \mathcal{C}_{a)}\right]+\mathcal{C}^{b} \nabla_{(a} \mathcal{C}_{b)}-\frac{1}{2} \gamma_{0} t_{a} \mathcal{C}^{b} \mathcal{C}_{b}
$$

This constraint evolution system has the same principal part as the unmodified system, equation (14). Therefore the same arguments about the self-consistency of the system and the preservation of the constraints within the domain of dependence apply. Similarly the relationship between the $\mathcal{C}_{a}$ constraint and the standard four-dimensional momentum constraint is not changed in any essential way: setting $\mathcal{C}_{a}=\partial_{t} \mathcal{C}_{a}=0$ on a $t=$ constant surface is still equivalent to setting $\mathcal{C}_{a}=\mathcal{M}_{a}=0$ there.

Consider the properties of the constraint evolution system for states that are very close to the constraint-satisfying submanifold $\mathcal{C}_{a}=\partial_{t} \mathcal{C}_{a}=0$. We can ignore the terms in equation (20) that are quadratic in $\mathcal{C}_{a}$ in this case, so the constraint evolution system reduces to

$$
0=\nabla^{b} \nabla_{b} \mathcal{C}_{a}-2 \gamma_{0} \nabla^{b}\left[t_{(b} \mathcal{C}_{a)}\right]
$$

Gundlach et al [15] have shown that all the 'short wavelength' solutions to this constraint evolution system are damped at either the rate $\mathrm{e}^{-\gamma_{0} t}$ or $\mathrm{e}^{-\gamma_{0} t / 2}$. This explains how the addition of the terms proportional to $\gamma_{0}$ in the modified GH system, equation (17), tend to damp out small constraint violations. This also explains (in part) why the numerical evolutions of this system by Pretorius were so successful. A complete understanding of how the long wavelength constraints are damped (or not) in generic spacetimes would also be quite interesting, but this is not yet fully understood.

\section{New first-order GH evolution system}

In this section we present a new first-order representation of the modified GH evolution system, which will (we think) be a useful counterpart to the second-order system described in section 2.2 above. There is an extensive mathematical literature on first-order evolution systems that clarifies numerous issues of great importance in numerical relativity, e.g., how to formulate well-posed boundary conditions [18-20], which systems form shocks [21], etc. We have also been more successful implementing first-order systems in our spectral evolution code.

The principal part of each component of the modified GH system, $\psi^{c d} \partial_{c} \partial_{d} \psi_{a b}$, is the same as the principal part of the covariant scalar-field system. So a first-order representation of the GH system can be constructed simply by adopting the methods used for scalar fields $[22,23]$. Using this method and the usual $3+1$ coordinates, equation (8), a first-order representation of the GH system can be written down, and indeed was written down (in essentially this form) by Alvi [24]:

$$
\begin{aligned}
& \partial_{t} \psi_{a b}-N^{k} \partial_{k} \psi_{a b} \simeq 0 \\
& \partial_{t} \Pi_{a b}-N^{k} \partial_{k} \Pi_{a b}+N g^{k i} \partial_{k} \Phi_{i a b} \simeq 0 \\
& \partial_{t} \Phi_{i a b}-N^{k} \partial_{k} \Phi_{i a b}+N \partial_{i} \Pi_{a b} \simeq 0
\end{aligned}
$$


where $\Phi_{i a b}=\partial_{i} \psi_{a b}$ and $\Pi_{a b}=-t^{c} \partial_{c} \psi_{a b}$ are new fields introduced to represent the first derivatives of $\psi_{a b}$. The notation $\simeq$ indicates that only the principal parts of the equations (i.e., the parts containing derivatives of the fields) are displayed.

In the discussion that follows, it will be helpful to discuss first-order evolution systems like this using a more compact and more abstract notation. Thus, we let $u^{\alpha}=\left\{\psi_{a b}, \Pi_{a b}, \Phi_{i a b}\right\}$ denote the collection of dynamical fields; and the evolution system for these fields can be written as

$$
\partial_{t} u^{\alpha}+A_{\beta}^{k \alpha} \partial_{k} u^{\beta}=F^{\alpha},
$$

where $A^{k \alpha}{ }_{\beta}$ and $F^{\alpha}$ may depend on $u^{\alpha}$ but not its derivatives. We use Greek indices throughout this paper to label the collection of dynamical fields. The principal part of this system is written abstractly as $\partial_{t} u^{\alpha}+A^{k \alpha}{ }_{\beta} \partial_{k} u^{\beta} \simeq 0$, so equations (22)-(24) determine the matrix $A^{k \alpha}{ }_{\beta}$ but not $F^{\alpha}$ for this system. First-order evolution systems of this form are called symmetric hyperbolic if there exists a symmetric positive definite matrix $S_{\alpha \beta}$ (the symmetrizer) on the space of fields that satisfies the condition $S_{\alpha \mu} A_{\beta}^{k \mu} \equiv A_{\alpha \beta}^{k}=A_{\beta \alpha}^{k}$. The mathematical literature on symmetric hyperbolic systems is extensive, and includes for example strong existence and uniqueness theorems [7, 18-20]. Alvi's representation of the GH system [24] is symmetric hyperbolic, as was a similar representation of the Einstein system (for the case of harmonic coordinates) given earlier by Fischer and Marsden [7].

Alvi's first-order representation of the GH system has two serious problems: first, the use of the field $\Phi_{i a b}$ introduces a new constraint,

$$
\mathcal{C}_{i a b}=\partial_{i} \psi_{a b}-\Phi_{i a b},
$$

which can (and does) tend to grow exponentially during numerical evolutions. Second, this system does not satisfy the mathematical condition (linear degeneracy) that prevents the formation of shocks from smooth initial data [21]. The principal part of the $\psi_{t i}$ component of equation (22), for example, can be written as $\partial_{t} N^{i}-N^{k} \partial_{k} N^{i} \simeq 0$; and these terms have the same form as those responsible for shock formation in the standard hydrodynamic equations.

We had previously developed ways to modify systems of this type to eliminate either of these problems [23]. However, these methods produce systems that are not symmetric hyperbolic when both problems are corrected simultaneously. Here we present new modifications that solve both problems without destroying symmetric hyperbolicity. We do this by adding appropriate multiples of the constraint $\mathcal{C}_{i a b}$ to each of the equations: $\gamma_{1} N^{i} \mathcal{C}_{i a b}$ to equation (22), $\gamma_{3} N^{i} \mathcal{C}_{i a b}$ to equation (23) and $\gamma_{2} N \mathcal{C}_{i a b}$ to equation (24). These terms modify the principal parts of the equations:

$$
\begin{aligned}
& \partial_{t} \psi_{a b}-\left(1+\gamma_{1}\right) N^{k} \partial_{k} \psi_{a b} \simeq 0 \\
& \partial_{t} \Pi_{a b}-N^{k} \partial_{k} \Pi_{a b}+N g^{k i} \partial_{k} \Phi_{i a b}-\gamma_{3} N^{k} \partial_{k} \psi_{a b} \simeq 0 \\
& \partial_{t} \Phi_{i a b}-N^{k} \partial_{k} \Phi_{i a b}+N \partial_{i} \Pi_{a b}-\gamma_{2} N \partial_{i} \psi_{a b} \simeq 0 .
\end{aligned}
$$

Choosing $\gamma_{3}=\gamma_{1} \gamma_{2}$ makes this new system symmetric hyperbolic for any values of the parameters $\gamma_{1}$ and $\gamma_{2}$. The symmetrizer metric (which defines the energy norm) for this new system can be written as

$$
S_{\alpha \beta} \mathrm{d} u^{\alpha} \mathrm{d} u^{\beta}=m^{a b} m^{c d}\left(\Lambda^{2} \mathrm{~d} \psi_{a c} \mathrm{~d} \psi_{b d}+\mathrm{d} \Pi_{a c} \mathrm{~d} \Pi_{b d}-2 \gamma_{2} \mathrm{~d} \psi_{a c} \mathrm{~d} \Pi_{b d}+g^{i j} \mathrm{~d} \Phi_{i a c} \mathrm{~d} \Phi_{j b d}\right),
$$

where $m^{a b}$ is any positive definite metric (e.g., $m^{a b}=g^{a b}+t^{a} t^{b}$ or even $m^{a b}=\delta^{a b}$ ) and $\Lambda$ is a constant with dimension length ${ }^{-1}$. This symmetrizer is positive definite so long as $\Lambda^{2}>\gamma_{2}^{2}$. 
The eigenvectors of the characteristic matrix, $n_{k} A_{\beta}^{k \alpha}$ (where $n_{k}$ is the outward directed unit normal to the boundary of the computational domain), play an important role in setting boundary conditions for first-order evolution systems. Let $e^{\hat{\alpha}}{ }_{\beta}$ denote the left eigenvectors with eigenvalues $v_{(\hat{\alpha})}$, defined by

$$
e^{\hat{\alpha}}{ }_{\mu} n_{k} A_{\beta}^{k \mu}=v_{(\hat{\alpha})} e_{\beta}^{\hat{\alpha}} \text {. }
$$

We use indices with hats (e.g., $\hat{\alpha}$ ) to label the characteristic eigenvectors and eigenvalues, and $\hat{\alpha}$ is not summed over in equation (31). The eigenvalues $v_{(\hat{\alpha})}$ are also called the characteristic speeds. The characteristic matrices of symmetric hyperbolic systems have complete sets of eigenvectors, so the matrix $e^{\hat{\alpha}}{ }_{\beta}$ is invertible in this case. The characteristic fields, $u^{\hat{\alpha}}$, are defined as the projections of the dynamical fields onto the characteristic eigenvectors: $u^{\hat{\alpha}} \equiv e^{\hat{\alpha}}{ }_{\beta} u^{\beta}$. Boundary conditions must be imposed on each incoming characteristic field, i.e., each $u^{\hat{\alpha}}$ with negative characteristic speed, $v_{(\hat{\alpha})}<0$ [18-20]. The characteristic fields for the new GH evolution system, equations (27)-(29), are given by

$$
\begin{aligned}
& u_{a b}^{\hat{0}}=\psi_{a b}, \\
& u_{a b}^{\hat{1} \pm}=\Pi_{a b} \pm n^{i} \Phi_{i a b}-\gamma_{2} \psi_{a b}, \\
& u_{i a b}^{\hat{2}}=P_{i}^{k} \Phi_{k a b},
\end{aligned}
$$

where $P_{i}{ }^{k}=\delta_{i}{ }^{k}-n_{i} n^{k}$. The characteristic fields $u_{a b}^{\hat{0}}$ have coordinate characteristic speed $-\left(1+\gamma_{1}\right) n_{k} N^{k}$, the fields $u_{a b}^{\hat{1} \pm}$ have speed $-n_{k} N^{k} \pm N$, and the fields $u_{i a b}^{\hat{2}}$ have speed $-n_{k} N^{k}$.

The complete equations for our new first-order representation of the GH evolution system (including all the non-principal parts) are

$$
\begin{aligned}
& \partial_{t} \psi_{a b}-\left(1+\gamma_{1}\right) N^{k} \partial_{k} \psi_{a b}=-N \Pi_{a b}-\gamma_{1} N^{i} \Phi_{i a b}, \\
& \partial_{t} \Pi_{a b}-N^{k} \partial_{k} \Pi_{a b}+N g^{k i} \partial_{k} \Phi_{i a b}-\gamma_{1} \gamma_{2} N^{k} \partial_{k} \psi_{a b} \\
&=2 N \psi^{c d}\left(g^{i j} \Phi_{i c a} \Phi_{j d b}-\Pi_{c a} \Pi_{d b}-\psi^{e f} \Gamma_{a c e} \Gamma_{b d f}\right) \\
& \quad-2 N \nabla_{(a} H_{b)}-\frac{1}{2} N t^{c} t^{d} \Pi_{c d} \Pi_{a b}-N t^{c} \Pi_{c i} g^{i j} \Phi_{j a b} \\
& \quad+N \gamma_{0}\left[2 \delta^{c}{ }_{(a} t_{b)}-\psi_{a b} t^{c}\right]\left(H_{c}+\Gamma_{c}\right)-\gamma_{1} \gamma_{2} N^{i} \Phi_{i a b}, \\
& \partial_{t} \Phi_{i a b}-N^{k} \partial_{k} \Phi_{i a b}+N \partial_{i} \Pi_{a b}-N \gamma_{2} \partial_{i} \psi_{a b} \\
&= \frac{1}{2} N t^{c} t^{d} \Phi_{i c d} \Pi_{a b}+N g^{j k} t^{c} \Phi_{i j c} \Phi_{k a b}-N \gamma_{2} \Phi_{i a b} .
\end{aligned}
$$

The terms on the right sides of equations (35)-(37) are algebraic functions of the dynamical fields. The connection terms $\Gamma_{c a b}$ appearing on the right side of equation (36) are computed using equations (3), where it is understood that the partial derivatives are to be determined from the dynamical fields by

$$
\begin{aligned}
& \partial_{t} \psi_{a b}=-N \Pi_{a b}+N^{i} \Phi_{i a b}, \\
& \partial_{i} \psi_{a b}=\Phi_{i a b} .
\end{aligned}
$$

Choosing the parameter $\gamma_{2}>0$ in this new system causes the constraint $\mathcal{C}_{i a b}$ to be exponentially suppressed [23], because the modified equation (37) implies an evolution equation for $\mathcal{C}_{i a b}$ having the form $\partial_{t} \mathcal{C}_{i a b}-N^{k} \partial_{k} \mathcal{C}_{i a b} \simeq-\gamma_{2} N \mathcal{C}_{i a b}$. Choosing $\gamma_{1}=-1$ makes the system equations (35)-(37) linearly degenerate, which implies that shocks do not form from smooth initial data [21]. And choosing the parameter $\gamma_{0}>0$ causes the constraint $\mathcal{C}_{a}$ to be exponentially suppressed, as discussed in section 2.2 . 


\section{Boundary conditions}

The modifications of the GH evolution system discussed in sections 2.2 and 3 are designed to damp out small constraint violations that may arise from inexact initial data, numerical errors, etc. These modifications will do nothing, however, to prevent the influx of constraint violations through the boundaries of the computational domain. Constraint-preserving boundary conditions are needed to prevent this [25-29]. Such boundary conditions can be formulated once the propagation equations for the constraints are understood. So we derive a first-order system of evolution equations for the constraints in section 4.1, use them to derive constraint-preserving boundary conditions in section 4.2, present boundary conditions for the physical gravitational-wave degrees of freedom of the system in section 4.3 , and finally analyse the well posedness of the combined set of new boundary conditions in section 4.4.

\subsection{First-order constraint evolution system}

The primary constraint of the $\mathrm{GH}$ system is the gauge constraint, $\mathcal{C}_{a}$, which we re-write here in terms of the first-order dynamical fields:

$$
\mathcal{C}_{a}=H_{a}+g^{i j} \Phi_{i j a}+t^{b} \Pi_{b a}-\frac{1}{2} g_{a}^{i} \psi^{b c} \Phi_{i b c}-\frac{1}{2} t_{a} \psi^{b c} \Pi_{b c}
$$

This expression differs from equation (12) only by multiples of the constraint $\mathcal{C}_{i a b}$. In the following we use this definition, equation (40), rather than equation (12), because it simplifies the form of the constraint evolution system. The evolution equation for $\mathcal{C}_{a}$, equation (14), is second order. Thus, we must define new constraint fields that represent the first derivatives of $\mathcal{C}_{a}$ in order to reduce the constraint evolution system to first-order form. Thus we define new constraint fields $\mathcal{F}_{a}$ and $\mathcal{C}_{i a}$ that satisfy

$$
\begin{aligned}
& \mathcal{F}_{a} \approx t^{c} \partial_{c} \mathcal{C}_{a}=N^{-1}\left(\partial_{t} \mathcal{C}_{a}-N^{i} \partial_{i} \mathcal{C}_{a}\right), \\
& \mathcal{C}_{i a} \approx \partial_{i} \mathcal{C}_{a},
\end{aligned}
$$

up to terms proportional to the constraints $\mathcal{C}_{a}$ and $\mathcal{C}_{i a b}$. The following definitions of $\mathcal{F}_{a}$ and $\mathcal{C}_{i a}$ accomplish this in a way that keeps the form of the constraint evolution system as simple as possible:

$$
\begin{aligned}
& \mathcal{F}_{a} \equiv \frac{1}{2} g_{a}^{i} \psi^{b c} \partial_{i} \Pi_{b c}-g^{i j} \partial_{i} \Pi_{j a}-g^{i j} t^{b} \partial_{i} \Phi_{j b a}+\frac{1}{2} t_{a} \psi^{b c} g^{i j} \partial_{i} \Phi_{j b c} \\
&+t_{a} g^{i j} \partial_{i} H_{j}+g_{a}^{i} \Phi_{i j b} g^{j k} \Phi_{k c d} \psi^{b d} t^{c}-\frac{1}{2} g_{a}^{i} \Phi_{i j b} g^{j k} \Phi_{k c d} \psi^{c d} t^{b} \\
&-g_{a}^{i} t^{b} \partial_{i} H_{b}+g^{i j} \Phi_{i c d} \Phi_{j b a} \psi^{b c} t^{d}-\frac{1}{2} t_{a} g^{i j} g^{m n} \Phi_{i m c} \Phi_{n j d} \psi^{c d} \\
&-\frac{1}{4} t_{a} g^{i j} \Phi_{i c d} \Phi_{j b e} \psi^{c b} \psi^{d e}+\frac{1}{4} t_{a} \Pi_{c d} \Pi_{b e} \psi^{c b} \psi^{d e}-g^{i j} H_{i} \Pi_{j a} \\
&-t^{b} g^{i j} \Pi_{b i} \Pi_{j a}-\frac{1}{4} g_{a}^{i} \Phi_{i c d} t^{c} t^{d} \Pi_{b e} \psi^{b e}+\frac{1}{2} t_{a} \Pi_{c d} \Pi_{b e} \psi^{c e} t^{d} t^{b} \\
&+g_{a}^{i} \Phi_{i c d} \Pi_{b e} t^{c} t^{b} \psi^{d e}-g^{i j} \Phi_{i b a} t^{b} \Pi_{j e} t^{e}-\frac{1}{2} g^{i j} \Phi_{i c d} t^{c} t^{d} \Pi_{j a} \\
&-g^{i j} H_{i} \Phi_{j b a} t^{b}+g_{a}^{i} \Phi_{i c d} H_{b} \psi^{b c} t^{d}+\gamma_{2}\left(g^{i d} \mathcal{C}_{i d a}-\frac{1}{2} g_{a}^{i} \psi^{c d} \mathcal{C}_{i c d}\right) \\
&+\frac{1}{2} t_{a} \Pi_{c d} \psi^{c d} H_{b} t^{b}-t_{a} g^{i j} \Phi_{i j c} H_{d} \psi^{c d}+\frac{1}{2} t_{a} g^{i j} H_{i} \Phi_{j c d} \psi^{c d}, \\
& \mathcal{C}_{i a} \equiv g^{j k} \partial_{j} \Phi_{i k a}-\frac{1}{2} g_{a}^{j} \psi^{c d} \partial_{j} \Phi_{i c d}+t^{b} \partial_{i} \Pi_{b a}-\frac{1}{2} t_{a} \psi^{c d} \partial_{i} \Pi_{c d} \\
&+\partial_{i} H_{a}+\frac{1}{2} g_{a}^{j} \Phi_{j c d} \Phi_{i e f} \psi^{c e} \psi^{d f}+\frac{1}{2} g^{j k} \Phi_{j c d} \Phi_{i k e} \psi^{c d} t^{e} t_{a} \\
&-g^{j k} g^{m n} \Phi_{j m a} \Phi_{i k n}+\frac{1}{2} \Phi_{i c d} \Pi_{b e} t_{a}\left(\psi^{c b} \psi^{d e}+\frac{1}{2} \psi^{b e} t^{c} t^{d}\right) \\
&-\Phi_{i c d} \Pi_{b a} t^{c}\left(\psi^{b d}+\frac{1}{2} t^{b} t^{d}\right)+\frac{1}{2} \gamma_{2}\left(t_{a} \psi^{c d}-2 \delta_{a}^{c} t^{d}\right) \mathcal{C}_{i c d} .
\end{aligned}
$$


The remaining constraints needed to complete the GH constraint evolution system are $\mathcal{C}_{i a b}$ defined in equation (26), and the closely related $\mathcal{C}_{i j a b}$, defined by

$$
\mathcal{C}_{i j a b}=2 \partial_{[i} \Phi_{j] a b}=2 \partial_{[j} \mathcal{C}_{i] a b} .
$$

The complete collection of constraints for the $\mathrm{GH}$ evolution system is therefore the set $c^{A} \equiv\left\{\mathcal{C}_{a}, \mathcal{F}_{a}, \mathcal{C}_{i a}, \mathcal{C}_{i a b}, \mathcal{C}_{i j a b}\right\}$ defined in equations (40), (43), (44), (26) and (45). (We use upper case Latin indices to label the constraints.) The constraints $c^{A}$ depend on the dynamical fields $u^{\alpha}=\left\{\psi_{a b}, \Pi_{a b}, \Phi_{i a b}\right\}$ and their spatial derivatives $\partial_{k} u^{\alpha}$. Thus the evolution of the constraint fields $c^{A}$ is completely determined by the evolution of the dynamical fields through equations (35)-(37). We have evaluated these constraint evolution equations and have verified that they can be written in the abstract form

$$
\partial_{t} c^{A}+A^{k A}{ }_{B}(u) \partial_{k} c^{B}=F^{A}{ }_{B}(u, \partial u) c^{B},
$$

where $A^{k A}{ }_{B}$ and $F^{A}{ }_{B}$ may depend on the dynamical fields $u^{\alpha}$ and their spatial derivatives $\partial_{k} u^{\alpha}$. Thus the constraint evolution system closes: the time derivatives of the constraints vanish initially when the constraints themselves vanish at an initial time. The principal part of the first-order constraint evolution system turns out to be remarkably simple (given the complexity of the expressions for the constraints themselves):

$$
\begin{aligned}
& \partial_{t} \mathcal{C}_{a} \simeq 0, \\
& \partial_{t} \mathcal{F}_{a} \simeq N^{i} \partial_{i} \mathcal{F}_{a}+N g^{i j} \partial_{i} \mathcal{C}_{j a}, \\
& \partial_{t} \mathcal{C}_{i a} \simeq N^{j} \partial_{j} \mathcal{C}_{i a}+N \partial_{i} \mathcal{F}_{a}, \\
& \partial_{t} \mathcal{C}_{i a b} \simeq\left(1+\gamma_{1}\right) N^{k} \partial_{k} \mathcal{C}_{i a b}, \\
& \partial_{t} \mathcal{C}_{i j a b} \simeq N^{k} \partial_{k} \mathcal{C}_{i j a b} .
\end{aligned}
$$

This constraint evolution system is symmetric hyperbolic with symmetrizer

$$
\begin{aligned}
S_{A B} \mathrm{~d} c^{A} \mathrm{~d} c^{B}= & m^{a b}\left[\mathrm{~d} \mathcal{F}_{a} \mathrm{~d} \mathcal{F}_{b}+g^{i j}\left(\mathrm{~d} \mathcal{C}_{i a} \mathrm{~d} \mathcal{C}_{j b}+g^{k l} m^{c d} \mathrm{~d} \mathcal{C}_{i k a c} \mathrm{~d} \mathcal{C}_{j l b d}\right)\right. \\
& \left.+\Lambda^{2}\left(\mathrm{dC}_{a} \mathrm{~d} \mathcal{C}_{b}+g^{i j} m^{c d} \mathrm{~d} \mathcal{C}_{i a c} \mathrm{~d} \mathcal{C}_{j b d}\right)\right],
\end{aligned}
$$

where $\Lambda^{2}$ is a positive constant and $m^{a b}$ is an arbitrary positive definite metric. The constraint energy for this system is defined as

$$
\mathcal{E}_{c}=\int S_{A B} c^{A} c^{B} \sqrt{g} \mathrm{~d}^{3} x .
$$

Since the constraint evolution system is hyperbolic, it follows (at the continuum level) that the constraints will remain satisfied within the domain of dependence of the initial data if they are satisfied initially.

We have analysed the solutions to this constraint evolution system for the case of small constraint violations of solutions near flat space. We find that all of the short-wavelength constraint violations are damped at the rate $\mathrm{e}^{-\gamma_{0} t}, \mathrm{e}^{-\gamma_{0} t / 2}$, or $\mathrm{e}^{-\gamma_{2} t}$. So choosing $\gamma_{0}>0$ and $\gamma_{2}>0$ is sufficient to guarantee that all of these constraints are suppressed. This new firstorder GH system therefore has the same constraint suppression properties as the second-order system of Gundlach et al [15] and Pretorius [14].

The constraint evolution system, equation (46), is symmetric hyperbolic and it will be useful to determine the characteristic constraint fields. Thus, we evaluate the matrix of left eigenvectors of the constraint evolution system $e^{\hat{A}}{ }_{B}$ and their corresponding eigenvalues $v_{(\hat{A})}$ (or characteristic speeds). The characteristic constraint fields are defined (in analogy with the principal evolution system) as the projections of the constraint fields onto these eigenvectors: 
$c^{\hat{A}} \equiv e^{\hat{A}}{ }_{B} c^{B}$. The resulting characteristic fields for this constraint evolution system are

$$
\begin{aligned}
& c_{a}^{\hat{0} \pm}=\mathcal{F}_{a} \mp n^{k} \mathcal{C}_{k a}, \\
& c_{a}^{\hat{1}}=\mathcal{C}_{a}, \\
& c_{i a}^{\hat{2}}=P^{k}{ }_{i} \mathcal{C}_{k a}, \\
& c_{i a b}^{\hat{3}}=\mathcal{C}_{i a b}, \\
& c_{i j a b}^{\hat{4}}=\mathcal{C}_{i j a b} .
\end{aligned}
$$

The characteristic constraint fields $c_{a}^{0 \pm}$ have coordinate characteristic speeds $-n_{l} N^{l} \pm N$, the fields $c_{a}^{\hat{1}}$ have speed 0 , the fields $c_{i a}^{\hat{2}}$ and $c_{i j a b}^{\hat{4}}$ have speed $-n_{l} N^{l}$ and the fields $c_{i a b}^{\hat{3}}$ have speed $-\left(1+\gamma_{1}\right) n_{l} N^{l}$.

\subsection{Constraint-preserving boundary conditions}

Boundary conditions must be imposed on all the incoming characteristic fields $u^{\hat{\alpha}}$, i.e., all those with $v_{(\hat{\alpha})}<0$ on a particular boundary. Thus, boundary conditions will typically be needed for the characteristic field $u_{a b}^{\hat{1}-}$, and (depending on the value of the parameter $\gamma_{1}$ and the orientation of the shift $N^{k}$ at the boundary) may also be needed for $u_{a b}^{\hat{0}}$ and/or $u_{i a b}^{\hat{2}}$. Some of these boundary conditions must be set by physical considerations, i.e., by specifying what physical gravitational waves enter the computational domain. Some of the boundary conditions can be used, however, to prevent the influx of constraint violations. This can be done by specifying the incoming $u^{\hat{\alpha}}$ at the boundary in a way that ensures the incoming characteristic constraint fields $c^{\hat{A}}$ also vanish there. The incoming constraint fields for this system include $c_{a}^{\hat{0}-}$, and perhaps $c_{i a b}^{\hat{3}}$ and/or $c_{i k a b}^{\hat{4}}$ depending on $\gamma_{1}$ and $N^{k}$ at the boundary. We find that these incoming $c^{\hat{A}}$ are related to the incoming $u^{\hat{\alpha}}$ by the following expressions:

$$
\begin{aligned}
& c_{a}^{\hat{0}-} \approx \sqrt{2}\left[k^{(c} \psi^{d)}{ }_{a}-\frac{1}{2} k_{a} \psi^{c d}\right] d_{\perp} u_{c d}^{\hat{1}-}, \\
& n^{i} c_{i a b}^{\hat{3}} \approx d_{\perp} u_{a b}^{\hat{0}}, \\
& n^{i} c_{i k a b}^{\hat{4}} \approx d_{\perp} u_{k a b}^{\hat{2}} .
\end{aligned}
$$

Here the notation $d_{\perp} u^{\hat{\alpha}}$ denotes the characteristic projection of the normal derivatives of $u^{\hat{\alpha}}$ (i.e., $d_{\perp} u^{\hat{\alpha}} \equiv e^{\hat{\alpha}} n^{k} \partial_{k} u^{\beta}$ ), and $\approx$ implies that algebraic terms and terms involving tangential derivatives of the fields (i.e., $P^{k}{ }_{i} \partial_{k} u^{\alpha}$ ) have not been displayed. The inward directed null vector $k^{c}$ used here is defined as $k^{c}=\left(t^{c}-n^{c}\right) / \sqrt{2}$. The idea is to set the left sides of equations (59)(61) to zero to get Neumann-like boundary conditions for the indicated components of $d_{\perp} u^{\hat{\alpha}}$. By imposing these conditions on $d_{\perp} u^{\hat{\alpha}}$, we ensure that these incoming components of $c^{\hat{A}}$ vanish.

We have found that a convenient way to impose boundary conditions of this type is to set the incoming projections of the time derivatives of $u^{\alpha}, d_{t} u^{\hat{\alpha}} \equiv e^{\hat{\alpha}}{ }_{\beta} \partial_{t} u^{\beta}$, in the following way:

$$
d_{t} u^{\hat{\alpha}}=D_{t} u^{\hat{\alpha}}+v_{(\hat{\alpha})}\left(d_{\perp} u^{\hat{\alpha}}-\left.d_{\perp} u^{\hat{\alpha}}\right|_{B C}\right) .
$$

In this expression the terms $D_{t} u^{\hat{\alpha}}$ represent the projections of the right sides of the evolution system, equations (35)-(37); so the equations at non-boundary points would simply be $d_{t} u^{\hat{\alpha}}=D_{t} u^{\hat{\alpha}}$. The term $\left.d_{\perp} u^{\hat{\alpha}}\right|_{B C}$ is the value to which $d_{\perp} u^{\hat{\alpha}}$ is to be fixed on the boundary. This form of the boundary condition replaces all of the $d_{\perp} u^{\hat{\alpha}}$ that appear in $D_{t} u^{\hat{\alpha}}$ with $\left.d_{\perp} u^{\hat{\alpha}}\right|_{B C}$. Applying this method to the constraint-preserving boundary conditions in equations (59)-(61), 
we obtain the following rather simple conditions

$d_{t} u_{a b}^{\hat{0}}=D_{t} u_{a b}^{\hat{0}}-\left(1+\gamma_{1}\right) n_{j} N^{j} n^{k} c_{k a b}^{\hat{3}}$,

$d_{t} u_{a b}^{\hat{1}-}=\left[\frac{1}{2} P_{a b} P^{c d}-2 l_{(a} P_{b)}{ }^{(c} k^{d)}+l_{a} l_{b} k^{c} k^{d}\right] D_{t} u_{c d}^{\hat{1}-}$

$$
+\sqrt{2}\left(N+n_{j} N^{j}\right)\left[l_{(a} P_{b)}{ }^{c}-\frac{1}{2} P_{a b} l^{c}-\frac{1}{2} l_{a} l_{b} k^{c}\right] c_{c}^{\hat{0}-},
$$

$d_{t} u_{k a b}^{\hat{2}}=D_{t} u_{k a b}^{\hat{2}}-n_{l} N^{l} n^{i} P^{j}{ }_{k} c_{i j a b}^{\hat{4}}$.

The quantity $P_{a b}$ in these expressions is the projection tensor, $P_{a b}=\psi_{a b}+t_{a} t_{b}-n_{a} n_{b}$, and the outgoing null vector $l^{a}$ is defined by $l^{a}=\left(t^{a}+n^{a}\right) / \sqrt{2}$.

\subsection{Physical boundary conditions}

The constraint-preserving boundary conditions presented in equations (63)-(65) restrict only four degrees of freedom of $u_{a b}^{\hat{1}-}$. Two of the remaining degrees of freedom represent the physical gravitational waves, and the final four represent gauge freedom. We choose to characterize and control this gravitational wave freedom in terms of the incoming parts of the Weyl curvature. The propagating components of the Weyl tensor can be written as

$$
w_{a b}^{ \pm}=\left(P_{a}^{c} P_{b}{ }^{d}-\frac{1}{2} P_{a b} P^{c d}\right)\left(t^{e} \mp n^{e}\right)\left(t^{f} \mp n^{f}\right) C_{c e d f} .
$$

We showed in [28] that these components of the Weyl tensor are the incoming and outgoing (respectively) characteristic fields of the curvature evolution system that follows from the Bianchi identities. The $w_{a b}^{ \pm}$are proportional to the Newman-Penrose curvature spinor components $\Psi_{4}$ (outgoing) and $\Psi_{0}$ (ingoing), respectively. We also note that the spatial components of $w_{i j}^{ \pm}$are equal to the components of the Weyl tensor characteristic fields $2 U_{i j}^{8 \pm}$ defined in our paper on constraint-preserving boundary conditions for the KST system [28]. The expression for the Weyl tensor in terms of our first-order variables is unique only up to terms proportional to constraints; it is possible to choose these constraint terms so that the $w_{i j}^{ \pm}$ depend on the normal derivatives of $u^{\hat{\alpha}}$ in the following way:

$$
w_{a b}^{ \pm} \approx\left(P_{a}^{c} P_{b}{ }^{d}-\frac{1}{2} P_{a b} P^{c d}\right)\left(d_{\perp} u_{c d}^{\hat{1} \pm}+\gamma_{2} d_{\perp} u_{c d}^{\hat{0}}\right) .
$$

Thus a physical boundary condition can be placed on the relevant components of $u_{a b}^{\hat{1}-}$ using the method of equation (62) by setting

$d_{t} u_{a b}^{\hat{1}-}=\left(P_{a}^{c} P_{b}{ }^{d}-\frac{1}{2} P_{a b} P^{c d}\right)\left[D_{t} u_{c d}^{\hat{1}-}-\left(N+n_{j} N^{j}\right)\left(w_{c d}^{-}-\gamma_{2} n^{i} c_{i c d}^{\hat{3}}\right)\right]$.

We can also inject incoming physical gravitational waves with a predetermined waveform $\dot{h}_{a b}(t, x)$ through the boundary of the computational domain by setting

$$
d_{t} u_{a b}^{\hat{1}-}=\left(P_{a}^{c} P_{b}{ }^{d}-\frac{1}{2} P_{a b} P^{c d}\right) \dot{h}_{c d}(t, x) .
$$

The case $\dot{h}_{a b}=0$ corresponds to an isolated system with no incoming gravitational waves.

More generally we can combine the constraint-preserving, physical no-incoming radiation, and the injected gravitational wave boundary conditions by setting $d_{t} u_{a b}^{\hat{1}-}$ equal to the sum of the right sides of equations (64), (68) and (69), and setting the time derivatives of the other incoming fields according to equations (63) and (65). Note that this set of combined boundary conditions holds the pure gauge components of $u_{a b}^{\hat{1}-}$ constant in time; other boundary conditions on the gauge degrees of freedom are of course possible but are not considered here. 

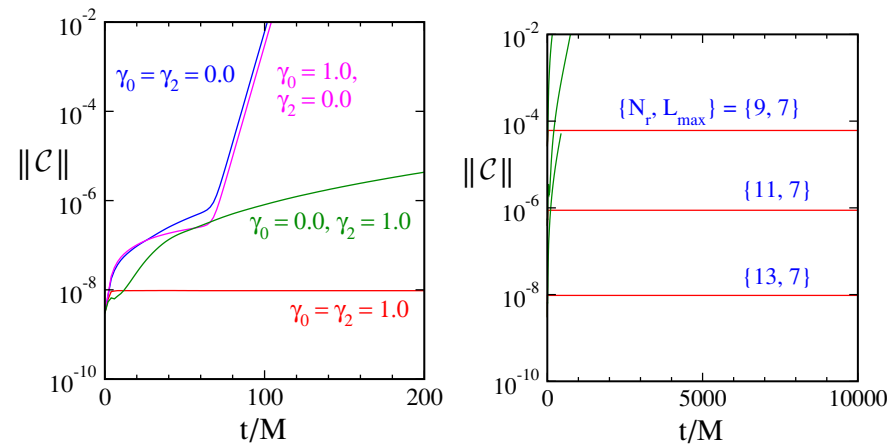

Figure 1. Evolution of constraint violations for Schwarzschild initial data. Left figure shows evolutions using various values of the constraint damping parameters $\gamma_{0}$ and $\gamma_{2}$ using numerical resolution $\left\{N_{r}, L_{\max }\right\}=\{13,7\}$. Right figure shows the long timescale evolution of the same data for three different numerical resolutions.

\subsection{Well posedness}

The well posedness of the initial-boundary value problem can be analysed using the FourierLaplace technique [30]. We have applied this method to the GH system with the combined set of boundary conditions presented here: we treat the case of high-frequency perturbations of flat spacetime in a slicing with flat spatial metric, unit lapse and a constant shift that is tangent to the boundary. Applying the Fourier-Laplace technique to this case yields a necessary (but not sufficient) condition for well posedness, the so-called determinant condition [30]; failure to satisfy this condition would mean the system admits exponentially growing solutions with arbitrarily large growth rates. We have verified that this determinant condition is satisfied for the GH system using the combined set of boundary conditions presented here.

\section{Numerical results}

In this section we describe several numerical tests of the new first-order GH evolution system. First we test the effectiveness of the two constraint damping terms included in equations (35)(37) by evolving Schwarzschild initial data (in Kerr-Schild coordinates). These tests are performed on a computational domain consisting of a spherical shell that extends from $r_{\min }=1.8 M$ (just inside the event horizon) to $r_{\max }=11.8 M$, where $M$ is the mass of the black hole. In these evolutions we 'freeze' the values of the incoming characteristic fields to their initial values by setting $d_{t} u^{\hat{\alpha}}=0$ on the boundaries for all incoming fields (i.e., all $u^{\hat{\alpha}}$ with $\left.v_{(\hat{\alpha})}<0\right)$. We performed these numerical evolutions using spectral methods as described for example in [28] for a range of numerical resolutions specified by the parameters $N_{r}$ (the highest radial spectral basis function) and $L_{\max }$ (the highest spherical-harmonic basis function). Figure 1 illustrates the results of these tests for several values of the constraint damping parameters $\gamma_{0}$ and $\gamma_{2}$. These tests show that without constraint damping the extended $\mathrm{GH}$ evolution system is extremely unstable, but with constraint damping the evolutions of the Schwarzschild spacetime are completely stable up to $t=10000 M$ (and forever, we presume). These tests also illustrate that both the $\gamma_{0}$ and the $\gamma_{2}$ constraint damping terms are essential for stable evolution.

Constraint violations in figure 1 (and in the rest of this paper) are measured with the constraint energy $\mathcal{E}_{c}$ defined in equation (53). Since $\mathcal{E}_{c}$ is not dimensionless, its magnitude 


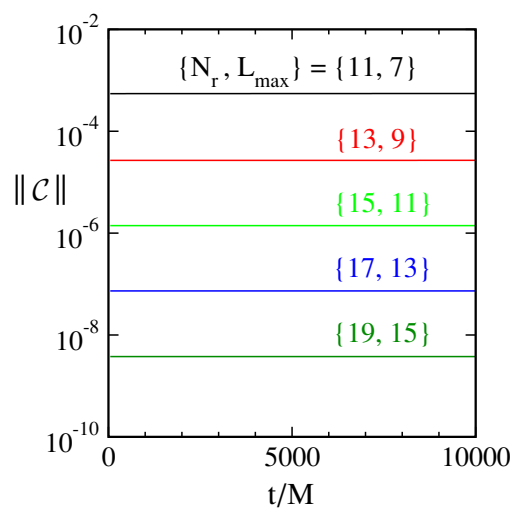

Figure 2. Evolution of constraint violations for Kerr initial data with spin parameter $\vec{a}=$ $(0.1,0.2,0.3)$ for several numerical resolutions.

has no absolute meaning. We construct an appropriate scale with which to compare $\mathcal{E}_{c}$ by evaluating the $L^{2}$ norm of the spatial gradients of the dynamical fields,

$\|\partial u\|^{2}=\int g^{i j} m^{a b} m^{c d}\left(\Lambda^{2} \partial_{i} \psi_{a c} \partial_{j} \psi_{b d}+\partial_{i} \Pi_{a c} \partial_{j} \Pi_{b d}+g^{k l} \partial_{i} \Phi_{k a c} \partial_{j} \Phi_{l b d}\right) \sqrt{g} \mathrm{~d}^{3} x$.

The dimensionless constraint norm $\|\mathcal{C}\|$ shown in these figures is defined as

$$
\|\mathcal{C}\|=\frac{\sqrt{\mathcal{E}_{c}}}{\|\partial u\|}
$$

which is a meaningful measure of the relative size of constraint violations in a particular solution. In the figures shown here we evaluate $\|\mathcal{C}\|$ with $m^{a b}=\delta^{a b}$ and the dimensional constant $\Lambda=1 / M$.

Our second numerical test evolves the somewhat more challenging initial data for a Kerr black hole (in Kerr-Schild coordinates) on a computational domain consisting of a spherical shell that extends from $r_{\min }=1.8 M$ (just inside the event horizon) to $r_{\max }=21.8 M$. We use two subdomains, each having numerical resolution $\left\{N_{r}, L_{\max }\right\}$, to cover this region. The spin of the Kerr spacetime used here is $\vec{a}=(0.1,0.2,0.3) M$, where the magnitude of this vector determines the Kerr spin parameter $a=|\vec{a}| \approx 0.374 M$, and the direction determines the orientation of the Kerr rotation axis relative to the quasi-Cartesian coordinate system used in our code. For this test we use the combined set of physical and constraint-preserving boundary conditions discussed at the end of section 4.3. Figure 2 shows that numerical evolutions of this Kerr spacetime are stable and numerically convergent to $t=10000 M$ (and forever, we presume) using a range of numerical resolutions.

Our third numerical test is designed to demonstrate the effectiveness of our new constraintpreserving boundary conditions. This test consists of evolving a black-hole spacetime perturbed by an incoming gravitational wave pulse. We start with Schwarzschild initial data, and perturb them via the incoming gravitational wave boundary condition described in equation (69) with $\dot{h}_{a b}=\dot{f}(t)\left(\hat{x}^{a} \hat{x}^{b}+\hat{y}^{a} \hat{y}^{b}-2 \hat{z}^{a} \hat{z}^{b}\right)$ where $\hat{x}^{a}, \hat{y}^{a}$ and $\hat{z}^{a}$ are the components of the coordinate basis vectors, $\hat{x}^{a} \partial_{a}=\partial_{x}$, etc. For these evolutions we use an incoming gravitational wave pulse whose time profile is $f(t)=\mathcal{A} \mathrm{e}^{-\left(t-t_{p}\right)^{2} / w^{2}}$ with $\mathcal{A}=10^{-3}, t_{p}=60 M$ and $w=10 M$. This test is performed on the same computational domain described above for the second numerical test. Figure 3 illustrates the results of these tests using two types of boundary conditions: frozen-incoming-field (i.e., $d_{t} u^{\hat{\alpha}}=0$ for $v_{(\hat{\alpha})}<0$ ) boundary conditions 

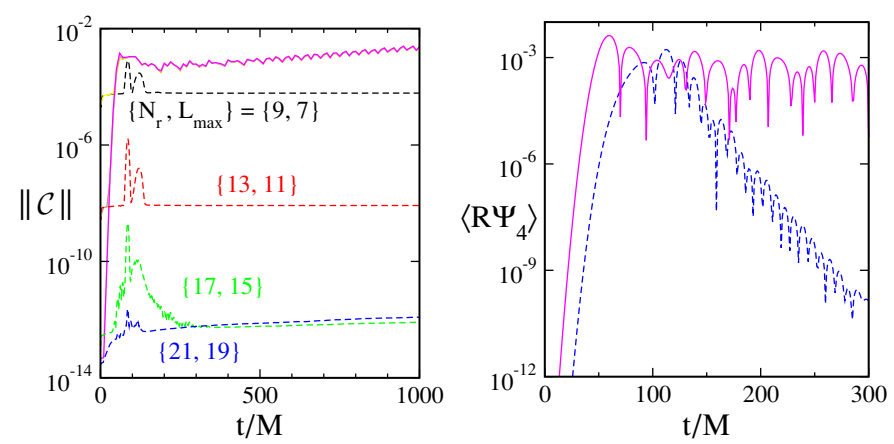

Figure 3. Evolution of Schwarzschild initial data perturbed by a gravitational wave pulse with amplitude $10^{-3}$. Left figure depicts constraint violations at various numerical resolutions, and the right figure shows $\Psi_{4}$ averaged over the outer boundary of the computational domain at a single numerical resolution. Solid curves use freezing boundary conditions and dashed curves use constraint-preserving and physical boundary conditions.

(solid curves) and the new combined set of constraint-preserving and physical boundary conditions discussed at the end of section 4.3 (dashed curves). The graph on the left in figure 3 shows that constraint violations converge towards zero as the numerical resolution is increased when the new boundary conditions are used, but not when frozen-incoming-field boundary conditions are used.

The graph on the right in figure 3 shows the outgoing physical gravitational wave flux (measured on the outer boundary of the computational domain) computed using frozen-incoming fields (solid curve) and the new constraint-preserving and physical (dashed curve) boundary conditions. These evolutions were computed with numerical resolution $\left\{N_{r}, L_{\max }\right\}=\{21,19\}$. We measure the outgoing gravitational wave flux with the quantity $\left\langle R \Psi_{4}\right\rangle$, which is the Weyl curvature component $\Psi_{4}$ averaged over the outer boundary of our computational domain:

$$
4 \pi\left\langle R \Psi_{4}\right\rangle^{2}=\int\left|\Psi_{4}\right|^{2} \mathrm{~d}^{2} V .
$$

Here $4 \pi R^{2}$ is the proper surface area of the boundary, and $\mathrm{d}^{2} V$ represents the proper area element on this boundary. Since $\Psi_{4}$ falls off like $1 / R$, this quantity should be independent of $R$ (asymptotically). The dashed curve on the right in figure 3 clearly shows quasi-normal oscillations with frequency $\omega M=0.376-0.089 \mathrm{i}$ (determined by a numerical fit to these data). This is in good agreement with the frequency of the most slowly damped quasi-normal mode of the black hole: $\omega M=0.37367-0.08896 \mathrm{i}$ [31]. It is interesting to note that the solid curve - using frozen-incoming-fields boundary conditions_-gives qualitatively incorrect results for the physical gravitational waveform, even though the level of constraint violations is fairly small numerically in this case. This is not surprising because the magnitude of constraint violations in this case is comparable to the size of the injected gravitational wave pulse.

\section{Acknowledgments}

We thank Steven Detweiler, Yvonne Choquet-Bruhat, Harald Pfeiffer, Frans Pretorius, Olivier Sarbach, Tilman Sauer, Saul Teukolsky and James York for helpful discussions concerning this work. LL thanks the Isaac Newton Institute for Mathematical Sciences for their hospitality 
during a visit in which a portion of this work was completed. This work was supported in part by a grant from the Sherman Fairchild Foundation to Caltech and Cornell, by NSF grants PHY-0099568, PHY-0244906 and NASA grants NAG5-10707, NAG5-12834 at Caltech, and by NSF grants PHY-0312072, PHY-0354631, and NASA grant NNG05GG51G at Cornell. Some of the computations for this project were performed with the Tungsten cluster at the National Center for Supercomputing Applications.

\section{References}

[1] Renn J and Sauer T 1998 Heuristics and mathematical representation in Einstein's search for a gravitational field equation History of General Relativity (Einstein Studies vol 7) ed H Goenner, J Renn and J Ritter (Cambridge, MA: Birkhäuser) pp 87-125

[2] DeDonder T 1921 La Gravifique Einsteinienne (Paris: Gauthier-Villars)

[3] DeDonder T 1927 The Mathematical Theory of Relativity (Cambridge, MA: Massachusetts Institute of Technology)

[4] Fock V 1959 The Theory of Space Time and Gravitation (New York: Pergamon)

[5] Fourès-Bruhat Y 1952 Théorème d'existence pour certains systèmes d'équations aux dérivées partielles non linéaires Acta Math. 88 141-225

[6] Bruhat Y 1967 Cauchy problem Gravitation: An Introduction to Current Research ed L Witten (New York: Wiley) pp 130-68

[7] Fischer A E and Marsden J E 1972 The Einstein evolution equations as a first-order quasi-linear symmetric hyperbolic system Commun. Math. Phys. 28 1-38

[8] Garfinkle D 2002 Harmonic coordinate method for simulating generic singularities Phys. Rev. D 65044029

[9] Szilágyi B, Schmidt B and Winicour J 2002 Boundary conditions in linearized harmonic gravity Phys. Rev. D 65064015

[10] Szilágyi B and Winicour J 2003 Well-posed initial-boundary evolution in general relativity Phys. Rev. D 68041501

[11] Babiuc M C, Szilágyi B and Winicour J 2006 Testing numerical evolution with the shifted gauge wave Class. Quantum Grav. 23 S321-43 (Preprint gr-qc/0511154)

[12] Friedrich H 1985 On the hyperbolicity of Einstein's and other gauge field equations Commun. Math. Phys. $100525-43$

[13] Pretorius F 2005 Numerical relativity using a generalized harmonic decomposition Class. Quantum Grav. 22 425-52

[14] Pretorius F 2005 Evolution of binary black hole spacetimes Phys. Rev. Lett. 95121101

[15] Gundlach C, Calabrese G, Hinder I and Martin-Garcia J M 2005 Constraint damping in the Z4 formulation and harmonic gauge Class. Quantum Grav. 22 3767-74

[16] Bona C, Ledvinka T, Palenzuela C and Žáček M 2003 General-covariant evolution formalism for numerical relativity Phys. Rev. D 67104005

[17] Friedrich H 2005 On the nonlinearity of the subsidiary systems Class. Quantum Grav. 22 L77

[18] Rauch J 1985 Symmetric positive systems with boundary characteristics of constant multiplicity Trans. Am. Math. Soc. 291 167-87

[19] Secchi P 1996 The initial boundary value problem for linear symmetric hyperbolic systems with characteristic boundary of constant multiplicity Diff. Integral Eqns 9 671-700

[20] Secchi P 1996 Well-posedness of characteristic symmetric hyperbolic systems Arch. Rat. Mech. Anal. 134 155-97

[21] Liu T P 1979 Development of singularities in the nonlinear waves for quasilinear hyperbolic partial differential equations J. Diff. Eqns 33 92-111

[22] Scheel M A, Erickcek A L, Burko L M, Kidder L E, Pfeiffer H P and Teukolsky S A 2004 3d simulations of linearized scalar fields in Kerr spacetime Phys. Rev. D 69104006

[23] Holst M, Lindblom L, Owen R, Pfeiffer H P, Scheel M A and Kidder L E 2004 Optimal constraint projection for hyperbolic evolution systems Phys. Rev. D 70084017

[24] Alvi K 2002 First-order symmetrizable hyperbolic formulations of Einstein's equations including lapse and shift as dynamical fields Class. Quantum Grav. 19 5153-62

[25] Stewart J M 1998 The Cauchy problem and the initial boundary value problem in numerical relativity Class. Quantum Grav. 15 2865-89

[26] Calabrese G, Lehner L and Tiglio M 2002 Constraint-preserving boundary conditions in numerical relativity Phys. Rev. D 65104031 
[27] Calabrese G, Pullin J, Sarbach O, Tiglio M and Reula O 2003 Well posed constraint-preserving boundary conditions for the linearized Einstein equations Commun. Math. Phys. 240 377-95

[28] Kidder L E, Lindblom L, Scheel M A, Buchman L T and Pfeiffer H P 2005 Boundary conditions for the Einstein evolution system Phys. Rev. D 71064020

[29] Sarbach O and Tiglio M 2005 Boundary conditions for Einstein's field equations: analytical and numerical analysis J. Hyperbolic Diff. Eqns 2 839-83

[30] Gustafsson B, Kreiss H-O and Oliger J 1995 Time Dependent Problems and Difference Methods (Pure and Applied Mathematics) (New York: Wiley)

[31] Chandrasekhar S and Detweiler S 1975 The quasi-normal modes of the Schwarzschild black hole Proc. R. Soc. Lond. A 344 441-52 